Service social

\title{
Les attitudes des membres à l'égard de leurs associations et coopératives
}

\section{Yvan Comeau}

Volume 44, numéro 1, 1995

Valeurs, pratiques, action sociale

URI : https://id.erudit.org/iderudit/706682ar

DOI : https://doi.org/10.7202/706682ar

Aller au sommaire du numéro

\section{Éditeur(s)}

École de service social de l'Université Laval

ISSN

1708-1734 (numérique)

Découvrir la revue

Citer cet article

Comeau, Y. (1995). Les attitudes des membres à l'égard de leurs associations et coopératives. Service social, 44(1), 95-114. https://doi.org/10.7202/706682ar
Résumé de l'article

Dans cet article, l'auteur présente une synthèse de deux recherches différentes ayant pour point commun les attitudes des membres à l'égard des associations et des coopératives. Malgré les particularités théoriques et méthodologiques de ces études, des convergences existent quant aux processus occasionnant des attitudes positives chez les membres. Il s'agit de : la démocratie, l'efficacité, la compétence, la culture de la solidarité, les affinités idéologiques, la sociabilité et l'attention à la vie quotidienne. Des dispositions organisationnelles favorables au développement d'attitudes positives chez les membres sont suggérées pour chacun de ces processus, notamment la présence de leaders, l'information, la consultation des membres, la saine gestion financière, la formation, la dissidence, l'attention aux interactions quotidiennes et les échanges confirmatifs (repas, fête, etc.). 


\section{Les attitudes des membres à l'égard de leurs associations et coopératives}

YVAN COMEAU Professeur Faculté des sciences de l'éducation Université Laval

Le présent article fait état de deux études originales* étalées sur six ans (1986 à 1992) et portant sur les attitudes de membres d'associations communautaires et de coopératives à l'égard de leur organisation. La première recherche concerne la participation aux associations communautaires et la seconde s'intéresse à la satisfaction dans les coopératives de travail. Après avoir rappelé les repères théoriques et méthodologiques de ces recherches, nous établirons les objets d'attitudes révélés par ces enquêtes. Nous expliquerons par la suite les principaux processus reliés à des attitudes favorables à l'implication des membres, c'est-à-dire la démocratie, l'efficacité, la compétence, la culture de la solidarité, les affinités idéologiques, la sociabilité et la vie quotidienne. Enfin, nous décrirons les dispositions organisationnelles qui leur sont associées : l'information, la consultation, la présence de leaders, la saine gestion financière, la formation, la dissidence, I'attention aux interactions et les échanges confirmatifs (repas, fêtes, etc.).

* Ces recherches ont été rendues possibles grâce à l'appui du Conseil de recherches en sciences humaines du Canada et de la Chaire de coopération Guy-Bernier de I'UQAM. 


\section{LES ÉTUDES EMPIRIQUES}

Lorsqu'il est question du concept d'attitude, les sciences sociales font référence à " un état dans lequel l'individu est prêt à répondre d'une certaine manière à une certaine stimulation» (Grawitz, 1990: 571).

Le terme [attitude] désigne une orientation des conduites ou des jugements, lorsque ceux-ci présentent une certaine cohérence et une certaine stabilité. C'est donc une variable inobservable, supposée sous-jacente à des conduites ou à des expressions verbales. Le terme désigne souvent une orientation générale, par exemple l'attitude antidémocratique (Boudon et al., 1989: 21).

Une attitude ne correspond donc pas à une action isolée, mais à un type de réactions qui se produit dans des circonstances semblables, et elle se distingue d'autres concepts comme la personnalité, les opinions ou les valeurs. Ainsi, les attitudes possèdent une certaine régularité, mais elles sont moins stables que les traits de la personnalité et moins fluctuantes que les opinions; les valeurs auxquelles un individu adhère sont globales, peu nombreuses et positives (seul le degré de désirabilité varie), alors que les attitudes renvoient à des objets particuliers, se comptent par milliers et sont bipolaires (positives et négatives) (Lafrenaye, 1994). Les attitudes, nous rappelle Grawitz, comprennent un "pôle caractérisation» (une prise de position) et un "pôle conduite» (des comportements). Toujours envisagée à l'égard d'un objet, une attitude comporte un aspect individuel et un aspect social dans la mesure où des influences extérieures interviennent.

On ne peut pas observer directement les attitudes, mais on les définit dans une recherche précise par ce qui est commun à un ensemble de prises de position et de comportements. En ce qui regarde les attitudes des membres à l'égard des associations, nous avons circonscrit deux objets de recherche - la participation et la satisfaction - permettant de connaître et de comprendre les processus sociaux et organisationnels associés aux attitudes positives et négatives.

La première étude, qui a pris la forme $d^{\prime}$ une thèse de doctorat (Comeau, 1990), porte sur la participation aux associations communautaires à Victoriaville. La participation est définie comme étant une activité sociale traduisant des attitudes positives envers une association, c'est-à-dire des réactions favorables à une implication soutenue. Dans cette recherche, nous nous intéressons au complexe de croyances et de comportements qui correspondent à la participation et à la 
non-participation ainsi qu'aux processus producteurs de ces attitudes, soit à des phénomènes organisationnels ou à des situations de la vie quotidienne. Cet objet de recherche et d'autres raisons, que nous énumérons maintenant, nous ont fait opter pour une sociologie de la vie quotidienne. Premièrement, cette approche de la participation permet de saisir la totalité et les rapports qu'ont entre eux les niveaux du quotidien (sociabilité, activités, appareils, groupes structurés et société globale) (Lefèbvre, 1961; 1968). C'est donc dire que différents aspects de l'activité associative interagissent à la fois avec la vie privée (sphère relative au logement, à la famille et aux personnes significatives) et la vie publique (sphère relative aux grandes organisations économiques, politiques et culturelles). Deuxièmement, la sociologie de la vie quotidienne porte un intérêt particulier à la subjectivité des individus (Douglas, 1980), ce qui constitue un élément de notre objet de recherche défini au début de ce paragraphe. Troisièmement, cette manière d'aborder le phénomène de la participation nous amène à considérer de manière multidimensionnelle les acteurs sociaux, de sorte que nous pouvons intégrer les dimensions des théories sur la participation aux associations (Jenkins, 1983), $c^{\prime}$ est-à-dire les théories de la privation (dimension politique), de la société de masse (dimension sociale) et de la mobilisation des ressources (dimension économique). Le tableau 1 offre une synthèse de ces théories dont la présentation complète demanderait évidemment un plus grand approfondissement.

\section{Tableau 1}

Synthèse des théories de la participation aux associations

\begin{tabular}{llll}
\hline Théorie & Synthèse & Auteur & Limite(s) de la théorie \\
\hline Privation & $\begin{array}{l}\text { Perte d'acquis suscitant un } \\
\text { mécontentement et incapacité } \\
\text { des autorités à maintenir les } \\
\text { institutions. }\end{array}$ & Gurr (1971) & $\begin{array}{l}\text { Théorie limitée } \\
\text { à l'amorce de l'action } \\
\text { collective. }\end{array}$ \\
$\begin{array}{llll}\text { Société } \\
\text { de masse }\end{array}$ & $\begin{array}{l}\text { L'industrialisation et l'urbani- } \\
\text { sation désorganisent les } \\
\text { communautés traditionnelles } \\
\text { et la société se caractérise } \\
\text { par l'anomie. }\end{array}$ & Kornhauser (1965) & $\begin{array}{l}\text { Théorie négligeant } \\
\text { la conscience des } \\
\text { acteurs sociaux et les } \\
\text { motifs d'association. }\end{array}$ \\
$\begin{array}{l}\text { Mobilisation } \\
\text { des ressources }\end{array}$ & $\begin{array}{l}\text { La participation est } \\
\text { une action rationnelle } \\
\text { en vue de satisfaire } \\
\text { des intérêts personnels. }\end{array}$ & Olson (1971) & $\begin{array}{l}\text { Éléments de contexte } \\
\text { mis à l'écart. }\end{array}$ \\
& Zald et Ash (1966) & $\begin{array}{l}\text { Postulat de la rationa- } \\
\text { lité des comporte- } \\
\text { ments économiques } \\
\text { discutable. }\end{array}$
\end{tabular}


Au cours de cette recherche, 27 personnes d'âge, de scolarité et de situation familiale variés, appartenant à une coopérative alimentaire, une association de sans-emploi, un syndicat en grève, une coopérative de travail et un organisme de solidarité internationale, ont fait pendant deux heures un récit spontané de leurs pratiques associatives dans toutes sortes d'organisations. Elles ont confirmé en grande partie le bien-fondé des théories du tableau précédent sur la participation, mais elles ont aussi enrichi notre compréhension de leurs attitudes envers les associations avec des catégories et des processus absents des théories usuelles, comme nous le verrons dans la prochaine section.

La deuxième étude concerne la satisfaction dans les coopératives de travail (Comeau, 1992). Les recherches sur la satisfaction au travail portent généralement sur des entreprises capitalistes, sauf exceptions (Greenberg, 1980, 1981; Oliver, 1984, 1987; RothschildWhitt et Whitt, 1986). D'un point de vue théorique, ces études s'inspirent soit des écoles physico-économique, des relations humaines ou des modèles de causalité (Locke, 1976) (tableau 2). Nous avons privilégié une perspective critique (Rioux, 1978) qui cherche à dépasser la simple appréciation du travail, pour nous intéresser à la capacité de l'organisation coopérative de susciter des attitudes positives envers l'établissement de rapports sociaux égalitaires au travail et l'instauration de la propriété sociale des moyens de production. Puisque les coopératives de travail se distinguent par la présence à la fois d'un "groupement de personnes» et d'une "entreprise» (Vienney, $1980: 13)$, cette recherche a révélé, chez les membres, des éléments originaux en matière d'attitudes à l'égard des associations. Pour recueillir les informations et permettre l'analyse de la satisfaction et de l'insatisfaction dans les coopératives de travail, 233 membres de 45 coopératives de travail ont répondu en 1990 à un questionnaire comportant des échelles d'attitudes. L'année suivante, 60 personnes ont été interviewées (20 en entrevues individuelles et 40 en entrevues de groupe), afin de nous permettre de comprendre le sens des attitudes révélées dans la description et l'inférence statistiques.

\section{LES OBJETS D'ATTITUDES CHEZ LES MEMBRES}

Les questionnaires remplis et les entrevues réalisées dans ces recherches nous ont donné accès à un ensemble considérable d'informations. Dans chacune de ces recherches, nous avons procédé à des regroupements du contenu sous forme de catégories à l'aide de 
Tableau 2

Écoles de la satisfaction au travail

\begin{tabular}{|c|c|c|c|}
\hline École & Synthèse & Auteur & Limite(s) de l'école \\
\hline \multirow[t]{3}{*}{$\begin{array}{l}\text { Physico- } \\
\text { économique }\end{array}$} & \multirow{3}{*}{$\begin{array}{l}\text { Une seule manière de faire } \\
\text { (one best way) déterminée par } \\
\text { un expert peut réduire la } \\
\text { fatigue, l'ennui et la monotonie } \\
\text { du travail. }\end{array}$} & \multirow[t]{3}{*}{ Taylor $(1957 ; 1911)$} & $\begin{array}{l}\text { Priorité accordée } \\
\text { à la productivité. }\end{array}$ \\
\hline & & & $\begin{array}{l}\text { Approche conduisant } \\
\text { à l'émiettement du } \\
\text { travail. }\end{array}$ \\
\hline & & & $\begin{array}{l}\text { Négation du savoir } \\
\text { ouvrier. }\end{array}$ \\
\hline $\begin{array}{l}\text { Relations } \\
\text { humaines }\end{array}$ & $\begin{array}{l}\text { Le type de supervision, } \\
\text { les relations dans l'entreprise } \\
\text { et le travail lui-même } \\
\text { déterminent la satisfaction. }\end{array}$ & $\begin{array}{l}\text { Mayo (1933) } \\
\text { Herzberg (1978) }\end{array}$ & $\begin{array}{l}\text { Peu de considération } \\
\text { pour la division sociale } \\
\text { du travail. }\end{array}$ \\
\hline \multirow[t]{2}{*}{$\begin{array}{l}\text { Modèles } \\
\text { de causalité }\end{array}$} & \multirow[t]{2}{*}{$\begin{array}{l}\text { Rapport statistique entre la } \\
\text { satisfaction et des variables } \\
\text { organisationnelles et sociales. }\end{array}$} & \multirow[t]{2}{*}{$\begin{array}{l}\text { Plusieurs milliers } \\
\text { de titres uniquement } \\
\text { aux États-Unis. }\end{array}$} & $\begin{array}{l}\text { Recherches } \\
\text { essentiellement } \\
\text { quantitatives. }\end{array}$ \\
\hline & & & $\begin{array}{l}\text { Études limitées à la } \\
\text { vérification des théories } \\
\text { (en particulier celle } \\
\text { d'Herzberg). }\end{array}$ \\
\hline
\end{tabular}

techniques rigoureuses d'analyse qualitative des données (Comeau, 1994). Les concepts et les processus qui suivent apparaissent tels quels dans les recherches, et nous proposons ici une mise en rapport inédite entre eux.

\section{La participation aux associations communautaires}

En ce qui concerne la participation aux associations communautaires, des objets d'attitudes sont proposés par Meister (1974:47-50), qui distingue trois formes de participation: la prise de responsabilités, l'appartenance aux groupements et l'utilisation des services. Pour notre part, nous avons relevé six thèmes sur la participation pour lesquels les membres manifestent des attitudes parfois positives, parfois négatives. Il s'agit de: la fondation, l'adhésion formelle, la présence aux instances décisionnelles, l'occupation d'un poste électif, les ressources matérielles et financières, la réalisation des tâches. Nous livrons dans les paragraphes suivants l'essentiel des objets $d^{\prime}$ attitudes vers lesquels convergent les témoignages des membres interviewés.

La fondation concerne la mise sur pied de l'association. C'est à partir d'une situation problématique jugée inacceptable que des 
leaders regroupent quelques personnes, $d^{\prime}$ après ce que nous révèlent les entrevues. Avec la formation d'un comité provisoire, nous assistons à la constitution du groupe initial qui met en place les premières dispositions matérielles généralement modestes et les règles temporaires de fonctionnement. Même embryonnaire, un tel regroupement s'oppose parfois aux institutions et son caractère inédit fait que les fondateurs doivent être tenaces, nous ont dit les personnes interviewées.

L'adhésion formelle désigne la période plus ou moins longue pendant laquelle les membres maintiennent ou non leur appartenance. Un consensus constaté chez les personnes interviewées veut que le point de départ de l'adhésion formelle soit une situation indésirable, voire oppressante (sexisme, exploitation, domination, exclusion, isolement, danger pour l'intégrité du corps, etc.), qui s'oppose à la réalisation des aspirations des sujets. En outre, la presque totalité des personnes rencontrées sont devenues membres d'une association parce qu'elles avaient été sollicitées de personne à personne, généralement par un acteur significatif comme un ami, un parent ou quelqu'un qu'elles estiment. Les personnes rencontrées ont indiqué plusieurs facteurs qui favorisent la poursuite de leur adhésion. Les associations qui suscitent, parmi les membres et la population, la crédibilité en raison de leur capacité de résoudre la situation indésirable, celles dont les orientations idéologiques sont conformes à celles des acteurs sociaux, qui sont démocratiques, qui permettent la sociabilité et la solidarité, et qui offrent un équilibre entre l'exigence de travail et les bénéfices que ces mêmes associations octroient, incitent les membres à poursuivre leur adhésion. Par contre, l'effet perturbateur de la vie publique (par exemple, obtenir un travail qui résout la situation indésirable ou déménager pour en occuper un), les exigences de la vie privée (soins aux enfants, production domestique, etc.), les différences idéologiques, le manque de démocratie, le sur-don de travail bénévole prolongé et le manque de solidarité sont de nature à conduire à la démission, nous ont répété plusieurs personnes interviewées.

La présence aux instances décisionnelles fait valoir le point de vue des membres sur les finalités des associations qui évoluent notamment parce que les situations indésirables changent. C'est pendant des réunions que les sujets ont l'occasion de s'exprimer collectivement sur les objectifs, le fonctionnement et la stratégie de l'association. Voter est le principal signe d'une décision démocratique formelle, nous disent les personnes rencontrées, mais de façon plus informelle les membres ressentent s'il existe dans l'association 
un esprit qui permet l'expression démocratique et une prise de décision autonome. L'autonomie de la prise de décision caractérise un processus démocratique, collectif et conscient qui suscite une attitude positive. À l'opposé, I'hétéronomie de la prise de décision définit un processus où les membres sont plus ou moins privés du pouvoir collectif de décision et elle suscite une attitude négative.

L'occupation d'un poste électif entraîne des attitudes à propos $d^{\prime}$ un titre particulier de responsabilité au sein de l'association, soit la présidence, la trésorerie, le secrétariat ou l'administration en général. Pour faire accepter le poste par une personne, les membres pratiquent la sollicitation. Ils disent solliciter des personnes qui ont des compétences particulières (formation scolaire, réalisations passées au sein de I'association, qualités personnelles, habiletés reconnues, intérêt pour un domaine d'activité de l'association et la jeunesse qui donne du dynamisme). À partir des entrevues, nous relevons quatre facteurs qui favorisent l'acceptation d'un poste: la promotion d'idées de ce qu'on croit être bon pour l'association et pour les membres, l'altruisme («aider les autres»), l'ancienneté qui procure un savoir concret de l'association et la confiance en soi ( $I^{\prime}$ aptitude de pouvoir s'affirmer dans les diverses situations de la vie quotidienne). La sollicitation échoue si les exigences de la vie privée et publique sont réelles, si les expériences passées ont été malheureuses et si les personnes sollicitées manquent de confiance en elles; à cet égard, le fait d'assumer une responsabilité peut favoriser l'acquisition d'une image positive de soi.

Pour continuer à assumer une responsabilité, les administrateurs élus ont besoin d'une bonne entente et $d^{\prime}$ 'un climat de camaraderie (la sociabilité et la solidarité) et l'autonomie collective de la prise de décision (la démocratie). Par ailleurs, les raisons d'abandonner un mandat avant son terme peuvent être: le manque de formation quant à la façon d'assumer la responsabilité, les différences idéologiques pour lesquelles les membres élus ne trouvent pas de compromis, le don de temps bénévole s'il est consenti de façon trop intense et soutenue, et les exigences de la vie privée et publique (un travail salarié qui nécessite beaucoup de récupération physique, par exemple).

Les ressources matérielles et financières sont un objet d'attitudes évoqué par les membres, puisque la résolution de la situation oppressante par une stratégie de services ou de contestation nécessite des ressources. Les programmes gouvernementaux contribuent dans la plus large mesure aux ressources des associations et les membres déplorent le caractère bureaucratique de la demande, les 
rapports à produire sur l'utilisation du financement, qui nécessitent un temps considérable, l'impression de subir des décisions arbitraires et la réticence à lutter contre l'État qui finance. Les gestes de solidarité entre les associations communautaires contribuent aussi à obtenir des ressources par les prêts d'argent, de locaux et la cohabitation. Le financement autonome (vendre des biens ou des services, tenir des manifestations culturelles et des activités-bénéfice) octroie également des ressources, mais ces campagnes nécessitent beaucoup d'efforts de la part des membres.

Les ressources des associations augmentent généralement avec leur pérennité et, pour les membres, c'est le local de l'association qui témoigne le mieux de cette évolution. L'augmentation des ressources donne l'impression aux membres qu'elle procure une reconnaissance accrue, facilite I'obtention de nouvelles ressources, améliore les chances de résoudre la situation oppressante et assure la reconnaissance publique. Lorsque les ressources diminuent, les membres et les responsables de l'association éprouvent de l'anxiété. Les salariés envisagent l'avenir avec angoisse et doivent limiter leurs aspirations personnelles. De façon générale, une baisse des ressources dans les associations signifie pour les adhérents une diminution des chances de résoudre les situations oppressantes.

La réalisation des tâches comprend I'ensemble des activités de production matérielle et symbolique accomplies par les membres dans les associations. II s'agit d'un don de temps de travail qui augmente lorsque les membres occupent un poste de responsabilité et qui varie selon la stratégie des associations. Pour faciliter la réalisation collective des tâches, les membres estiment nécessaires l'existence de traits organisationnels et le respect de certaines attitudes cognitives des membres. Les caractères organisationnels qui favorisent la réalisation des tâches sont la perception d'une bonne coordination (la "bonne administration", le "bon jugement» et le sens pratique des responsables), les équipes nombreuses (qui limitent la tâche de chaque membre et qui montrent que l'association est bien vivante), les tâches valorisantes et les rituels (moment prévu et régulier de la contribution personnelle). La présence de certaines attitudes cognitives dans I'association peut favoriser la réalisation des tâches. La formation, les possibilités de faire ses propres apprentissages et la ténacité comptent parmi les principaux éléments cognitifs mentionnés par les sujets pour faciliter le travail collectif. Par ailleurs, les membres désignent les phénomènes qui ne favorisent pas la réalisation des tâches: des manières d'agir inappropriées (par exemple, appeler les membres à la dernière minute pour effectuer des 
tâches), les exigences de la vie privée et publique, les tâches trop lourdes et l'accaparement par les salariés des tâches de l'association.

\section{La satisfaction dans les coopératives de travail}

Les travaux de Herzberg (1978) nous ont permis de mettre en évidence quelques objets d'attitudes en matière de satisfaction dans les coopératives de travail: la politique de l'entreprise, le travail proprement dit, les relations avec les sociétaires, la supervision et les aspects financiers. Nous avons ajouté le mode de gestion, le soutien public et la vie privée pour tenir compte du matériel fourni par les entrevues.

Le mode de gestion vise la distribution du pouvoir dans la coopérative, le processus concret de la prise de décision, l'accès à l'information et les différents avantages de la forme de propriété. Les entrevues et les questionnaires montrent que la démocratie suscite une attitude positive chez les coopératrices et coopérateurs du travail. D'ailleurs, nous avons établi un lien significatif entre le fait d'avoir occupé un poste électif ou une responsabilité quelconque dans la coopérative et le niveau de satisfaction. Contrairement à ce qui a cours dans les entreprises capitalistes, c'est la capacité d'élire les membres du conseil d'administration qui, lui, embauche le directeur général, et la possibilité d'avoir accès à toute l'information touchant les aspects financiers de l'entreprise qui réjouissent les travailleurs. Par contre, les membres déplorent plus ouvertement le manque de compétence des administrateurs ou des sociétaires qui connaissent mal le fonctionnement de la coopérative en tant qu'association ou entreprise. Dans les questionnaires et dans les entrevues, les membres affirment que la propriété coopérative crée un sentiment d'appropriation de l'entreprise qui les incite à participer à son fonctionnement et à offrir un produit de qualité.

Les rapports avec les sociétaires concernent l'appréciation accordée aux membres, la manière dont se règlent les conflits et l'esprit collectif. Les répondants au questionnaire situent la qualité des relations entre les membres au sommet de leur satisfaction dans les coopératives de travail. On comprend mieux pourquoi dans les entrevues: c'est l'implication des autres sociétaires, leur intérêt pour I'entreprise et leur contribution à l'amélioration de l'entreprise qui suscitent une attitude positive envers les collègues. À l'opposé, les membres qui montrent de la résistance aux mesures d'adaptation de la coopérative, ceux qui ne prennent pas d'initiative, les individualistes, les personnes qui s'imposent et celles qui accordent la priorité à l'aspect salarial irritent les coopérateurs. De façon générale, 
les distances qui se créent entre les membres suscitent du désappointement. La production dans des départements différents, les statuts auxquels sont rattachés des privilèges et une mauvaise intégration des nouveaux, notamment, s'accompagnent d'attitudes négatives à l'égard de l'entreprise collective. Ces phénomènes s'observent principalement dans les coopératives de grande taille, et $c^{\prime}$ est pourquoi il existe une relation statistique entre la taille de la coopérative et le niveau de satisfaction générale.

Le travail proprement dit touche la division du travail, le matériel et les outils, le secteur d'activité de la coopérative et divers aspects relatifs aux tâches, c'est-à-dire les horaires de travail, le travail concret, la présence de défis à relever, etc. L'entraide au travail et la diversification des tâches suscitent du plaisir chez les travailleurs des entreprises collectives, alors que la division technique et sociale du travail, de même qu'une trop grande spécialisation des tâches, leur déplaît. La responsabilité individuelle ou l'autonomie au travail provoque un grand contentement. II arrive souvent aussi que le secteur d'activité de l'entreprise plaise aux membres.

La politique de l'entreprise concerne les finalités que celle-ci se fixe, l'idéologie qui l'anime et ses aspects culturels. La forme de propriété coopérative séduit les travailleurs qui l'expérimentent, dans la mesure où la participation financière va de pair avec une participation aux décisions. Nous avons observé un lien statistique entre le fait qu'une coopérative soit mise sur pied par les travailleurs eux-mêmes et un niveau de satisfaction élevé, sans doute à cause des efforts que cette mise sur pied exige. Aussi, une grande majorité des répondants au questionnaire sont reconnaissants aux coopératives de travail de leur procurer un emploi et s'estiment heureux que les producteurs soient au centre des préoccupations de ces entreprises.

Les aspects financiers portent sur la situation financière des coopératives de travail et sur les conditions de travail. Dans les questionnaires, les sociétaires situent au dernier rang ces éléments en matière de satisfaction. II semble que la crainte d'un dérapage financier soit constante, d'autant plus que les sociétaires sont informés de tous les aspects de la performance économique de leur entreprise. L'appréciation des salaires dépend du secteur économique des coopératives de travail, des aspirations des sociétaires en matière de niveau de vie et de leur conception du travail.

Le mode de supervision correspond au contrôle et à l'évaluation du travail, à la reconnaissance des réalisations et à l'espace réservé à la créativité. Généralement, les membres sont satisfaits du contrôle qu'ils exercent sur leur travail, dans la mesure où ils peuvent 
déterminer eux-mêmes collectivement les mesures d'évaluation du travail, reconnaissant ainsi l'importance de l'imputabilité. Mais il arrive que les contremaîtres aient des comportements dominateurs, ce qui évidemment déplaît aux sociétaires. Les coopératives qui offrent aux travailleurs la possibilité de proposer de nouvelles idées pour produire mieux ou pour diversifier la production font régner une ambiance agréable. Les superviseurs qui bloquent I'initiative des travailleurs hypothèquent l'implication des travailleurs.

La prise en compte de la vie privée par l'entreprise collective suscite des attitudes positives, surtout lorsque des liens d'amitié se sont établis pendant la fondation de la coopérative de travail. Il est plus facile pour les membres de petites organisations de se rencontrer à l'extérieur du travail et d'en arriver à connaître leurs familles respectives.

Le soutien du public en général et de l'État en particulier constitue une déception pour les coopératrices et les coopérateurs du travail. En effet, la population ne connaît pas la coopération du travail et les membres sentent peu d'encouragement à continuer, surtout lorsqu'on s'approche des grands centres urbains, sans doute parce que les coopératives sont moins intégrées à la dynamique locale du développement. Enfin, les administrateurs des coopératives de travail déplorent l'attitude tiède de certaines institutions financières à leur égard.

\section{LES DISPOSITIONS ORGANISATIONNELLES FAVORISANT DES ATTITUDES POSITIVES}

Nous venons de voir les objets $d^{\prime}$ attitudes mentionnés dans deux recherches différentes portant sur les associations et les coopératives de travail, chaque étude s'inspirant de traditions théoriques différentes. Il s'agit en somme de thèmes à l'égard desquels les membres de ces organisations manifestent des attitudes positives et négatives. À des fins de synthèse et de formalisation théorique, nous réduisons les processus associés aux attitudes positives et évoquées précédemment à sept notions essentielles pour favoriser l'acquisition d'attitudes positives par les membres: la démocratie, l'efficacité, la compétence, la culture de la solidarité, les affinités idéologiques, la sociabilité et I'attention à la vie privée. Pour chaque notion, nous relevons quelques dispositions organisationnelles susceptibles de favoriser des attitudes positives chez les membres et ce, à partir de l'expérience des organisations visitées et des écrits sur le sujet. 
La démocratie désigne un principe organisationnel selon lequel les membres exercent la souveraineté et le pouvoir. Nous avons vu, dans les paragraphes sur l'adhésion formelle, la présence aux instances décisionnelles, l'occupation d'un poste électif et le mode de gestion, que la notion de démocratie est au centre des attitudes des membres dans la mesure où ce pouvoir de décision peut conduire à des changements que les individus estiment significatifs (Gruneberg, 1981 : 88). On distingue généralement la démocratie directe ou participative, et la démocratie représentative. La forme participative encourage la formation d'un consensus large, alors que la forme représentative assure une qualité des décisions. La forme directe le groupe "assemblé et délibérant» dit Rosanvallon (1976:64) convient mieux aux petites organisations, alors que la forme élective répond mieux à la réalité des grands ensembles (Godbout, 1991). En termes organisationnels, la démocratie peut être encouragée par les mesures suivantes: la présence massive des membres, l'information, l'existence d'un contre-pouvoir, la consultation malgré la grande taille de l'organisation et les procédures délibérantes adéquates.

Pour susciter des attitudes positives, l'organisation démocratique doit, premièrement, rechercher la présence du plus grand nombre en soumettant à la discussion et aux prises de décision des thèmes importants pour les membres, qui touchent leur situation et qui les incitent à se rendre aux rencontres. Deuxièmement, l'information joue un rôle essentiel pour démocratiser le processus de décision. Pour Meister (1974 : 97-98), l'information encourage la participation, car elle suscite des attitudes favorables au changement. Lorsque l'information devient abondante, il faut la coder, c'est-à-dire la résumer et la rendre compréhensible à l'ensemble des membres, sans pour autant appauvrir le message (Lemoigne et Carré, 1977 : 37-38). Troisièmement, le contre-pouvoir renvoie à la présence d'une instance, d'un comité ou encore d'un syndicat, qui permet d'éviter le pouvoir arbitraire d'une autre forme d'autorité. Quatrièmement, dans les grandes organisations communautaires et coopératives, la démocratie ne peut pas se limiter à la représentation. Certaines personnes craignent la croissance des organisations, de peur que la démocratie directe ne perde de sa vitalité (Conforth et Thomas, 1990 : 456). On peut créer dans les grandes organisations des groupes de travail qui poursuivent à leur manière les objectifs de production des corps décisionnels et consultatifs intermédiaires, une rotation des tâches (Rothschild et Russell, 1986) ou une autonomie relative par secteurs géographiques ou autres. Cinquièmement, lorsque les membres, et en particulier les leaders, maîtrisent plus ou moins bien les techniques d'animation ou connaissent mal les implications concrètes de la 
démocratie, les membres risquent d'éprouver du ressentiment à l'égard de la prise de décision. Nous observons ce phénomène surtout dans les coopératives récentes où I'expérience fait défaut. Cette dynamique renvoie à la notion de compétence que nous aborderons plus loin.

L'efficacité désigne la capacité de l'organisation d'atteindre ses objectifs avec la plus grande économie possible de moyens. On se rappelle que l'efficacité suscitait des attitudes positives en matière d'adhésion, de ressources, d'aspects financiers et de réalisation des tâches. Nous relions trois domaines à l'efficacité : la stratégie, la coordination et la gestion financière.

Premièrement, la stratégie est entendue ici comme étant la combinaison de l'ensemble des moyens mis en œuvre pour résoudre la situation oppressante. Les membres contribuent inégalement à la stratégie puisque, affirme Bourdet (1976), seuls certains perçoivent clairement les objectifs. Évidemment, I'association exige des réajustements continuels et des adaptations constantes pour atteindre les objectifs. À cet égard, le leader fonctionnel a un rôle important à jouer, car c'est lui qui se préoccupe de la tâche et de l'action, qui propose des idées nouvelles, qui invite à l'efficacité et qui rappelle régulièrement les objectifs; pour sa part, le leader affectif favorise les relations humaines et l'harmonie. Deuxièmement, l'efficacité passe par la coordination du travail et des tâches. Celle-ci se réalise collectivement par divers outils de planification adaptés à la culture des membres (Ampleman et al., 1983; 1987). Les tâches doivent être partagées équitablement et il faut établir leur rotation. L'attitude des salariés doit être responsabilisante pour les membres. Dans certaines associations, la ritualisation de tâches (savoir qu'à tel moment du mois, par exemple, il y a telle corvée) favorise leur exécution. Troisièmement, en ce qui a trait à la gestion financière, nous avons vu que les membres des coopératives de travail suivent de près l'évolution financière. Pour les associations et les coopératives, la rentabilité détermine si les opérations peuvent se poursuivre. Dans le cas des coopératives de travail, les sociétaires souhaitent conserver leur emploi et, si possible, accroître leur marge bénéficiaire et utiliser les surplus sous forme de dividendes ou d'investissement dans I'entreprise, selon les limites prescrites par la Loi sur les coopératives. Même si les coopératives de travail sont aussi efficaces que des entreprises capitalistes comparables (Defourny, 1990), les membres ne sont jamais complètement satisfaits de la situation financière (Oliver, 1987; Rothschild-Whitt et Whitt, 1986). C'est pourquoi les mesures suivantes de saine gestion financière suscitent des attitudes positives: le recours à des instruments comptables, les prévisions 
budgétaires rigoureuses et les décisions permettant de réaliser des économies.

D'autres dispositions administratives peuvent remplir le même rôle: la recherche de la qualité du produit ou du service afin de garder les clients et surpasser les concurrents, I'appel à des ressources extérieures spécialisées, l'intercoopération, l'acquisition d'équipements, l'investissement dans la recherche pour la hausse de la productivité, la diversification des activités, l'intégration verticale au niveau des matières premières et de la commercialisation, et, surtout, le temps de travail bénévole qui fait partie de plusieurs contrats d'adhésion. Le réinvestissement dans la coopérative fait également partie des mesures qui recueillent l'assentiment des membres. En ce qui a trait aux risques de l'investissement, le comportement et la satisfaction des sociétaires se comparent à ceux des entrepreneurs privés. On s'assure de la sécurité des placements et on apprécie le fait d'investir de façon calculée dans l'entreprise, afin d'assurer le succès financier de la coopérative.

Un dernier mot sur la gestion financière porte sur le financement étatique, dont les associations notamment ne doivent pas dépendre totalement. Elles doivent elles aussi opérer une saine gestion financière, développer la solidarité avec d'autres groupes et procéder à des campagnes de financement autonome. Nous pensons qu'un niveau de financement trop élevé nuit à certains groupes qui ne peuvent pas absorber une croissance trop rapide et il menace leur militantisme; par ailleurs, un niveau trop faible des ressources paralyse I'action.

La compétence désigne la connaissance théorique et pratique permettant $d^{\prime}$ exercer une fonction, ainsi que les qualités professionnelles et administratives des membres. Nous savons maintenant que la compétence entraîne des attitudes positives pour les aspects suivants: I'adhésion, l'occupation d'un poste électif, la réalisation des tâches, le mode de gestion, les rapports avec les sociétaires, le mode de supervision, le travail proprement dit et les aspects financiers. Même si la reconnaissance de notre compétence chez les autres suscite la satisfaction, il faut rappeler, comme le propose l'analyse stratégique, qu'une compétence difficilement remplaçable constitue une des sources de pouvoir (Bernoux, 1985 : 132-135). La compétence s'acquiert principalement par l'expérience et la formation. En ce qui concerne la formation, il ressort que les coopératives de travail recourent presque exclusivement à la formation sur le tas. D'autres coopératives systématisent davantage la formation. Par exemple, certaines $d^{\prime}$ entre elles offrent une formation sur mesure pour les nouveaux membres. Ailleurs, chaque rencontre débute par une période 
de formation. D'autres coopératives proposent une formation basée sur des thèmes précis: le règlement des conflits, le français de base, les mathématiques, etc. Dans des occasions particulières, on met en place des activités spéciales de formation; dans des difficultés, on assiste à des activités de créativité (brassage d'idées ou brainstorming). Comprendre l'importance de la formation dans les associations et désirer la promouvoir pose la question de sa pratique pédagogique. Un champ extrêmement vaste s'ouvre ici et nous ne pouvons que formuler certains principes. Moins les membres sont scolarisés, plus les codes doivent être visuels, le langage concret, l'animation dynamique et les outils pédagogiques variés. La classe populaire maîtrise peu l'écrit et la graphie doit tenir compte de ce trait culturel. Puisque les personnes qui assistent à la formation occupent de cette façon une période normalement dévolue aux loisirs, le ludisme et la fête s'intègrent à la formation, sans pour autant que les formateurs négligent la préparation rigoureuse des activités éducatives. Pour effacer les mauvais souvenirs de l'école, l'imagination pédagogique doit être mise à profit afin que dans l'avenir les membres veuillent bien poursuivre leur formation.

La culture de la solidarité concerne l'intérêt des membres pour le groupe et l'intérêt commun, de même que l'esprit collectif basé sur le partage de certaines valeurs et normes. En d'autres termes, la solidarité est "la reconnaissance mutuelle que s'accordent des acteurs sociaux s'identifiant à un même ensemble" (notre traduction de Melucci, 1989 : 29). Il s'agit d'un caractère général des relations entre les membres qui reconnaissent la mutualité de leurs intérêts et qui se portent spontanément assistance. La solidarité est associée à des attitudes positives, surtout lorsqu'il est question de la fondation, de l'adhésion, de l'occupation d'un poste électif, des ressources matérielles et financières, de la réalisation des tâches, des rapports avec les sociétaires, du travail proprement dit et du soutien du public.

Nous proposons l'hypothèse que la solidarité se construit jour après jour, par des pratiques et des méthodes, plus ou moins explicites, qui se réalisent dans des interactions visant à faire valoir le bien commun. L'observation de ces méthodes pourrait conduire à élaborer une ethnométhodologie de la solidarité, c'est-à-dire une connaissance «des méthodes que les individus utilisent pour donner sens et en même temps accomplir leurs actions de tous les jours: communiquer, prendre des décisions, raisonner » (Coulon, $1987: 26)$ et développer un esprit collectif. Voici une série de méthodes quotidiennes que nous avons observées dans une association et qui permettent la constitution d'une culture de la solidarité : 
- se rencontrer régulièrement et mieux connaître les autres;

- aplanir les différences idéologiques en analysant collectivement des situations;

- prôner l'utilisation du "nous » et du «on » plutôt que du "vous » lorsqu'il est question du groupe;

- partager un vocabulaire commun ;

- construire un espoir collectif en se donnant un but explicite;

- parler de la solidarité : «il faut se donner la main»;

- sensibiliser au bien commun : "c'est notre affaire»;

- reprendre les commentaires qui ne vont pas dans le sens de la solidarité.

La culture de la solidarité suppose qu'un grand nombre de membres constituent l'association. Nous avons vu que la sollicitation incite les individus à se présenter aux activités de l'association. Nous considérons que cette incitation permet un apprentissage non intentionnel (Peterson, 1979 cité par Blais, 1983: 12) qui peut être combiné à d'autres processus éducatifs du même ordre, comme l'observation, l'imitation, la socialisation ou l'expérimentation individuelle.

Les affinités idéologiques permettent aux membres de déterminer dans quelle mesure l'analyse de la situation-problème, les objectifs et la stratégie de l'association sont conformes à leurs propres valeurs et idéologie. Nous savons que les affinités idéologiques sont à la source d'attitudes positives en matière d'adhésion, d'occupation d'un poste électif et de politique de l'entreprise. Les sujets peuvent poursuivre leur participation même s'ils perçoivent des différences idéologiques, dans la mesure où leur dissidence est exprimée et prise en considération par les autres membres. $D^{\prime}$ après notre recherche, ce sont les personnes scolarisées qui accordent le plus d'importance à la dimension idéologique. Cela est confirmé par une autre recherche portant sur la participation syndicale des cols blancs (Nicholson et al., 1981). Dans un grand nombre de coopératives, la dimension idéologique ne préoccupe pas les membres qui ne ressentent pas la nécessité d'exprimer une vision du monde et de la faire prévaloir. Nous posons l'hypothèse que le secteur d'activité peut rendre nécessaire l'explicitation d'une perception du monde, comme dans les coopératives du secteur culturel et social.

La sociabilité, définie comme recherche perpétuelle de la présence et de l'échange matériel et symbolique avec d'autres acteurs sociaux, est empreinte d'affectivité. Elle concerne particulièrement I'adhésion, l'occupation d'un poste électif et les rapports avec les 
sociétaires. La sociabilité est possible par les manières de convenance, c'est-à-dire les procédés par lesquels les sujets assurent la poursuite de cette sociabilité. Nous identifions en particulier les échanges confirmatifs (salutations, présentation des personnes, fête, repas, etc.). C'est souvent l'équipe de travail qu'on associe à une "famille», puisque sont présentes la confiance, la sociabilité et l'affection.

$\mathrm{L}^{\prime}$ attention à la vie quotidienne permet aux associations de surmonter l'opposition entre la vie associative et la vie privée, particulièrement aiguë pour les femmes qui assument encore aujourd'hui une grande part de la production domestique et qui doivent résister au pouvoir du conjoint sur leurs activités associatives. Comme le confirme une recherche menée auprès des femmes (Martin et Hanson, 1985), la flexibilité des horaires convient aux membres qui doivent s'occuper des enfants. II arrive que des mesures financières ou des activités récréatives touchent directement les ménages, ce qui plaît bien sûr aux membres. D'autres mesures indiquent que les coopératives accordent une attention à la vie privée. Les conditions de travail qui tiennent compte du nombre de personnes dans le ménage, les activités récréatives, la flexibilité des horaires et les programmes de santé sont des mesures qui ont fait l'objet d'une grande appréciation au cours des entrevues.

\section{CONCLUSION}

Partagé par plusieurs dirigeants d'associations et de coopératives, le souci de développer chez les membres des attitudes favorables à l'égard de leur organisation nous a interpellé et incité à entreprendre des recherches. C'est avec un souci de synthèse que nous avons présenté les résultats de deux recherches partageant un même intérêt: les attitudes des membres. Ces recherches, réalisées à des moments différents, ont chacune leur objet de recherche - la participation aux associations, d'une part, et la satisfaction dans les coopératives de travail, d'autre part -, leurs propres théories de référence, une opération distincte de collecte des données et des catégories spécifiques $d^{\prime}$ analyse de contenu. Toutefois, elles s'inspirent de méthodes semblables d'analyse qualitative des données. Celles-ci favorisent la " découverte» et des recherches statistiques seraient nécessaires pour permettre la "vérification» des résultats auprès d'un très grand nombre d'individus et d'associations et pour prétendre à une certaine universalité. 
Nous avons relevé certains phénomènes à l'égard desquels les membres manifestent différentes attitudes, sans prétendre épuiser la complexité du phénomène associatif. En ce qui a trait à la participation dans les associations, nous avons montré que la fondation, I'adhésion formelle, l'occupation d'un poste électif, les ressources matérielles et financières ainsi que la réalisation des tâches suscitent des attitudes tantôt positives, tantôt négatives, selon certains processus organisationnels. II en est de même en ce qui concerne la satisfaction dans les coopératives de travail. Le mode de gestion, les rapports avec les sociétaires, le travail proprement dit, la politique de l'entreprise, les aspects financiers, le mode de supervision, la prise en considération de la vie et le soutien du public soulèvent également des réactions diverses chez les sociétaires.

Nous avons établi les convergences suivantes entre ces études. II ressort que des processus fondamentaux sont associés à ces attitudes et qu'il est possible pour les dirigeants d'associations et $d^{\prime}$ 'entreprises collectives d'instituer des dispositions organisationnelles pouvant occasionner des attitudes positives. Nous avons nommé ces processus: la démocratie, l'efficacité, la compétence, la culture de la solidarité, les affinités idéologiques, la sociabilité et l'attention à la vie quotidienne. Parmi toutes les dispositions organisationnelles favorables à l'acquisition d'attitudes positives, nous retenons: I'information, la consultation, la présence de leaders, la saine gestion financière, la formation, la dissidence, l'attention aux interactions quotidiennes et les échanges confirmatifs (repas, fêtes, etc.).

Au terme de cette synthèse et de cette réflexion, il nous semble important pour les responsables de garder à l'esprit que les groupes communautaires et les coopératives sont à la fois des entreprises et des associations. Dans l'organisation sociétale occidentale et contemporaine, ces organisations peuvent être considérées comme des communautés permettant d'établir des ponts entre l'individu atomisé dans sa vie privée et la société hétéronome sur laquelle celui-ci n'a pas de prise. En ce sens, nous définissons la vie associative comme étant une praxis collective organisée et totalisante inscrite dans la vie quotidienne et visant la résolution de situations indésirables par I'instauration de communautés modernes. Nous les désignons ainsi, car elles amalgament la proximité sociale des communautés traditionnelles et une démarche rationnelle visant I'atteinte de certains objectifs. 


\section{Références bibliographiques}

Ampleman, G. et al. (1987). Pratiques de conscientisation 2. Québec: Collectif québécois d'édition populaire.

Ampleman, G. et al. (1983). Pratiques de conscientisation. Montréal: Nouvelle Optique.

BERNOUX, P. (1985). La sociologie des organisations. Paris: Seuil.

BLAIS, M. (1983). Les typologies en éducation des adultes: reflets d'une réalité en évolution. Montréal: Université de Montréal.

Boudon, R., P. Besnard, M. Cherkaoul et B.-P. LÉCuyer (1989). Dictionnaire de la sociologie. Paris : Larousse.

BOURDET, Y. (1976). Qu'est-ce qui fait courir les militants? Paris : Éditions Stock.

BRAUN, D.D. (1976). "Alienation and Participation : A Replication Comparing Leaders and the 'Mass' ", Journal of Political and Military Sociology, vol. $4: 245-259$.

COMEAU, Y. (1994). L'analyse des données qualitatives. Montréal : Collectif de recherche sur les innovations sociales dans les entreprises et les syndicats, cahier $\mathrm{n}^{\circ} 0294$.

COMEAU, Y. (1992).Les coopératives de travail au Québec et la satisfaction des membres. Chaire de coopération Guy-Bernier de I'UQAM, cahier $\mathrm{n}^{\circ}$ 02092-044.

COMEAU, Y. (1990) Vie quotidienne et participation aux associations en milieu populaire. Thèse de doctorat, Département de sociologie, Université de Montréal.

CONFORTH, C. et A. ThOMAS (1990). "Cooperative Development : Barriers, Support Structures and Cultural Factors », Economic and Industrial Democracy, vol. $11: 451-461$.

Coulon, A. (1987). L'ethnométhodologie. Paris: PUF, Que sais-je?

DEFOURNY, J. (1990). Démocratie coopérative et efficacité économique. Bruxelles: De Boeck/Wesmael.

Douglas, J. (dir.) (1980). Introduction to the Sociologies of Everyday Life. Boston: Allyn and Bacon.

DupuY, J.-P. (1982). Ordres et désordres. Paris: Seuil.

GODBOUT, J.T. (1991). "La participation politique : leçons des dernières décennies», dans J.T. Godbout, La participation politique. Québec: IQRC, Coll. Questions de culture, p. 11-31.

Grawitz, M. (1990). Méthodes des sciences sociales. Paris: Dalloz.

GREENBERG, E.S. (1981). "Industrial Self-Management and Political Attitudes », The American Political Science Review, n० 75 : 29-42.

GREENBERG, E.S. (1980). "Participation in Industrial Decision-Making and Work Satisfaction: The Case of the Producer Cooperatives », Social Science Quarterly, vol. 60:551-559.

GRUNEBERG, M.M. (1981). Understanding Job Satisfaction. London/Basingstoke: The Macmillan Press.

GURR, T.R. (1971). Why Men Rebel. Princeton: Princeton University Press. 
HerzberG, F. (1978). Le travail et la nature de l'homme. Paris: Entreprise Moderne d'Édition.

JENKINS, J.C. (1983). "Resource Mobilization Theory and the Study of Social Movements ", Annual Review of Sociology, vol. $9: 527-553$.

KORNhAusER, W. (1965). The Politics of Mass Society. Londres: Routledge and Kegan Paul.

LAFRENAYE, Y. (1994). "Les attitudes et le changement des attitudes", dans R.J. Vallerand (dir.), Les fondements de la psychologie sociale. Boucherville: Gaëtan Morin éditeur, p. 327-405.

LEFÈBVRE, H. (1961). Critique de la vie quotidienne II: Fondements d'une sociologie de la quotidienneté. Paris: L'Arche.

LEFÈBVRE, H. (1968). La vie quotidienne dans le monde moderne. Paris: Gallimard.

LEMOIGNe, J.-LouIS et D. CARRÉ (1977). Auto-organisation de l'entreprise. 50 propositions pour l'autogestion. Paris : Les Éditions d'organisation.

LOCKE, E.A. (1976). "The Nature and Causes of Job Satisfaction", dans M.D. Dunnette (dir.), Handbook of Industrial and Organizational Psychology. Chicago: Rand McNally College Publishing Company, p. 1297 1349.

MAYO, E. (1933). The Social Problems of an Industrial Civilization. Boston: Harvard University.

MeIsTeR, A. (1974). La participation dans les associations. Paris: Éditions ouvrières.

MELUCCI, A. (1989). Nomads of The Present. Londres: Radius.

NICHOLSON, N., G. URSELL et J. LUBBOCK (1981). "Membership Participation in a White-Collar Union", Industrial Relations, vol. 20, $\mathrm{n}^{\circ} 2: 162-178$.

OLIVER, N. (1987). "Commitment and Satisfaction in Producer Co-operatives: the Role of Work Values », The Journal of Interdisciplinary Economics, vol. 2 : 117-130.

OlIVER, N. (1984). "An Examination of Organizational Commitment in Six Worker's Cooperatives in Scotland», Human Relations, vol. 37, $\mathrm{n}^{\circ} 1$ : 29-46.

OLSON, M. (1971). The Logic of Collective Action. Cambridge: Harvard University Press.

RIOUX, M. (1978). Essai de sociologie critique. Montréal: Hurtubise HMH. Rosanvallon, Pierre (1976). L'âge de l'autogestion. Paris: Seuil.

ROTHSCHILD, J. et R. RUSSELL (1986). "Alternatives to Bureaucracy: Democratic Participation in the Economy », Annual Review of Sociology, vol. 12 : 307-328.

ROTHSCHILD-WHITT, J. et J.A. WHITT (1986). "Workers-owners as an Emergent Class : Effects of Cooperative Work on Job Satisfaction, Alienation and Stress", Economic and Industrial Democracy, vol. 7 : 297-317.

TAYLOR, F.W. (1957). La direction scientifique des entreprises. Paris: Dunod.

VIENNEY, C. (1980). Socio-économie des organisations coopératives. Paris: Coopérative d'information et d'édition mutualiste.

ZALD, M.N. et R. ASH (1966). "Social Movement Organizations : Growth, Decay and Change», Social Forces, vol. 44, n' $3: 327-341$. 